Case Report

\title{
Rhabdomyolysis: An Unusual Presentation of Mycoplasma pneumoniae Infection in an Adult-A Case Report and Literature Review
}

\author{
Jaspreet Kaler $\mathbb{D}^{1},{ }^{1}$ Osama Mukhtar $\mathbb{D}^{1},{ }^{1}$ Bilal Khan, ${ }^{1}$ Binav Shrestha $\mathbb{D}^{1}{ }^{1}$ \\ Ravinder Kaler, ${ }^{2}$ Brandon Ting, ${ }^{3}$ and Mazin Khalid ${ }^{1}$ \\ ${ }^{1}$ Department of Medicine, Interfaith Medical Center, Brooklyn, NY, USA \\ ${ }^{2}$ Caribbean Medical University, Curacao, Netherlands \\ ${ }^{3}$ Avalon University School of Medicine, Curacao, Netherlands
}

Correspondence should be addressed to Jaspreet Kaler; jessiekaler01@gmail.com

Received 17 February 2018; Accepted 29 April 2018; Published 21 June 2018

Academic Editor: David Grimaldi

Copyright ( $\odot 2018$ Jaspreet Kaler et al. This is an open access article distributed under the Creative Commons Attribution License, which permits unrestricted use, distribution, and reproduction in any medium, provided the original work is properly cited.

Mycoplasma pneumoniae is a common cause of community-acquired pneumonia, and many extrapulmonary manifestations have been described, but rhabdomyolysis is infrequently reported in adults. Of the few cases that have been reported in adults, it was almost exclusively seen when pneumonia was present. We report a case of a 30-year-old male who came in with complaints of fever and myalgia for three days. Immunoglobulin $\mathrm{M}$ antibodies for Mycoplasma pneumoniae were positive and trending up, despite having no radiographic evidence of pneumonia on chest X-ray or CT scan. He was treated successfully with levofloxacin and intravenous hydration. Later, his condition was clinically and biochemically improved, and he was discharged. Our patient did not present with typical respiratory tract symptoms of a mycoplasma infection. In addition, there was an absence of pneumonia on imaging, suggesting that rhabdomyolysis secondary to mycoplasma might be underdiagnosed and go untreated in the setting of low clinical suspicion. Upon review of the literature, there is only one other case of mycoplasma infection where rhabdomyolysis occurred in the absence of pneumonia. However, the degree of rhabdomyolysis in our case was much more severe. Although rare, when faced with rhabdomyolysis, Mycoplasma pneumoniae should be kept as a differential diagnosis even in the absence of pneumonia on radiological imaging.

\section{Introduction}

Mycoplasma pneumoniae is a rod-shaped bacterium, which affects $1 \%$ of the United States (US) population annually [1]. It is one of the most common causes of atypical pneumonia in the US, and it is transmitted from person to person via respiratory droplets. This disease has an incubation period of 2-3 weeks and can affect people of any age group but is most commonly found in school-aged children and military and college students. When infected, patients can present with clinical symptoms or be completely asymptomatic. Typical symptoms include headache, malaise, fever, chills, and cough. In fact, $75-100 \%$ of infected patients have an intractable nonproductive cough and 3-10\% of infected patients develop pneumonia [2]. Extrapulmonary disease occurs in less than
$5-10 \%$ of patients infected with mycoplasma [3]. This can include hematological, dermatological, gastrointestinal, neurological, and musculoskeletal manifestations. Of the several musculoskeletal manifestations, rhabdomyolysis has rarely been seen in Mycoplasma pneumoniae. In the cases that have been reported, rhabdomyolysis usually occurs in the pediatric population. We present an unusual case of Mycoplasma pneumoniae infection presenting with rhabdomyolysis in an adult patient where no radiographic evidence of pneumonia was detected.

\section{Case Presentation}

A 30-year-old male, with no significant past medical history, came to the emergency department with complaints of fever 
and chills for a duration of 3 days. He also complained of myalgia and abdominal pain for the same duration. $\mathrm{He}$ reported diffuse abdominal pain, which was $6 / 10$ in severity, nonradiating, and not related to food. He also reported 2 episodes of diarrhea without any blood or mucous, brown in color. He denied cough, shortness of breath, chest pain, night sweats, weight loss, history of trauma, extreme exercise, or any history of travel.

His abdominal examination was significant for abdominal tenderness in all four quadrants, but was soft and nondistended and bowel sounds were present. The rest of his examination was unremarkable. Triage vitals were significant for a temperature of $103.3^{\circ} \mathrm{F}$, heart rate of 88 beats per minute, respiratory rate of 18 breaths per minute, blood pressure of $109 / 49$, and pulse oximetry of $99 \%$ on room air.

Admission laboratory workup revealed leukocyte count of $6.1 \times 10^{3} \mu \mathrm{L}$ (reference range $4.5-11.0 \times 10^{3}$ ) with $72 \%$ neutrophils, hemoglobin of $16.2 \mathrm{~g} / \mathrm{dL}$ (reference range 13.0-17.0), hematocrit of $48.2 \%$ (reference range 39-53), platelet count of $149 \times 10^{3} \mu \mathrm{L}$ (reference range $130-400 \times 10^{3}$ ), ESR of $4 \mathrm{~mm} / \mathrm{hr}$ (reference range $0-20$ ), BUN of $34 \mathrm{mg} / \mathrm{dL}$ (reference range $8-20$ ), creatinine of $1.8 \mathrm{mg} / \mathrm{dL}$ (reference range $0.4-1.3$ ), potassium of $4.7 \mathrm{mmol} / \mathrm{L}$ (reference range 3.6-5.1), phosphorous of $3.0 \mathrm{mg} / \mathrm{dL}$ (reference range 2.4-4.7), aspartate aminotransferase of 1,146 IU/L (reference range 15-41), alanine aminotransferase of $243 \mathrm{IU} / \mathrm{L}$ (reference range 17-63), and creatinine kinase of 39,125 IU/L (reference range 38-398). Urinalysis was significant for large blood, and urine red blood cells were $0-2$.

In view of the high-grade fever, we attempted to find an infectious source to explain his history and presentation. Chest X-ray (Figure 1) and CT scan were obtained and showed no signs of pneumonia. Urinalysis and influenza rapid antigen test were performed and did not reveal any abnormalities. CT of the abdomen and pelvis was obtained and did not reveal any focal sources. Stool culture with ova and parasites, blood cultures, urinary toxicology including synthetic THC (K2), HIV, herpes simplex virus, hepatitis panel, Mycoplasma pneumoniae IgM antibody, urine Legionella antigen, QuantiFERON ${ }^{\circledR}$ test for tuberculosis, and TSH were all performed as well. All the test results were negative except immunoglobulin $\mathrm{M}$ antibodies for $\mathrm{Myco-}$ plasma pneumoniae that were detected by enzyme-linked immunosorbent assay. The antibodies were initially $781 \mathrm{U} / \mathrm{mL}$, which is considered a low positive (reference range 770-950).

Our patient was empirically started on levofloxacin and metronidazole for possible gastroenteritis initially while culture and laboratory results were pending. He was managed for his acute renal failure and rhabdomyolysis with aggressive parenteral hydration. However, he continued to spike fevers during days 1-4 of the admission, but his diarrhea, abdominal pain, and myalgia resolved by day 3 . One week later, mycoplasma antibody was recorded again and it was $976 \mathrm{U} / \mathrm{ml}$, which is considered a true positive (reference range $950+$ ). The fact that the antibody titer was trending up was clinically significant for a recent Mycoplasma pneumoniae infection. After mycoplasma was found to be positive, metronidazole was discontinued and he was continued

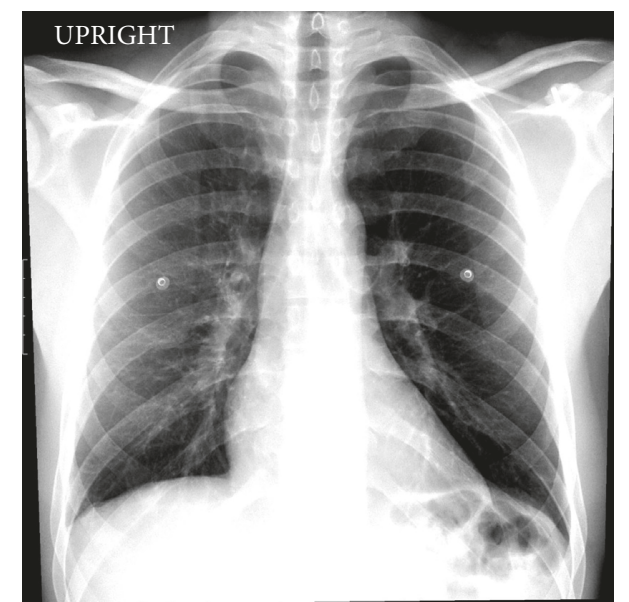

Figure 1: Chest X-ray showing no evidence of acute lung pathology.

with levofloxacin. His creatinine, liver function tests, and CK trended down to normal reference ranges (Figure 2), and he was discharged on day 9 with planned outpatient follow-up.

\section{Discussion}

The pathophysiology of rhabdomyolysis is based upon muscle necrosis, resulting in a release of intracellular components. This results in an increase of creatinine kinase and myoglobin in urine. Clinically, rhabdomyolysis can be asymptomatic or it can present with myalgia and lead to renal failure with electrolyte derangements. There are a variety of triggers that result in rhabdomyolysis, which includes trauma, extreme exertion, metabolic myopathy, thermal extremes, hypothyroidism, medications, drugs (alcohol, heroin, cocaine, amphetamine, methadone, D-lysergic acid diethylamide), electrolyte disorders (hypokalemia, hypophosphatemia), and certain infections. Infections account for less than $5 \%$ of all rhabdomyolysis causes [4]. Infectious causes include influenza A and B, coxsackievirus, Epstein-Barr virus, herpes simplex, parainfluenza, adenovirus, echovirus, enterovirus, human immunodeficiency virus, cytomegalovirus, varicella-zoster, Mycoplasma pneumoniae, Legionella pneumophila, tularemia, Streptococcus pneumoniae, Salmonella, E. coli, leptospirosis, Coxiella burnetii, Staphylococcus, ehrlichiosis, falciparum malaria, Clostridium perfringens, and Chlamydia psittaci [5-19]. This patient's history, clinical data, rising titers of mycoplasma antibodies, and his clinical response to levofloxacin are all compatible with Mycoplasma pneumoniae being the trigger for rhabdomyolysis.

Rhabdomyolysis triggered by Mycoplasma pneumoniae is a rare extrapulmonary manifestation in adults. Our case is unique since there was no radiographic evidence of pneumonia. Review of literature has shown that the earliest case of rhabdomyolysis in an adult with Mycoplasma pneumoniae was reported as early as 1979 [20]. To our knowledge, 7 cases of Mycoplasma pneumoniae with rhabdomyolysis have been described in adults since then (Table 1) [20-26]. Of the reported cases, Decaux et al. reported the one other case, 


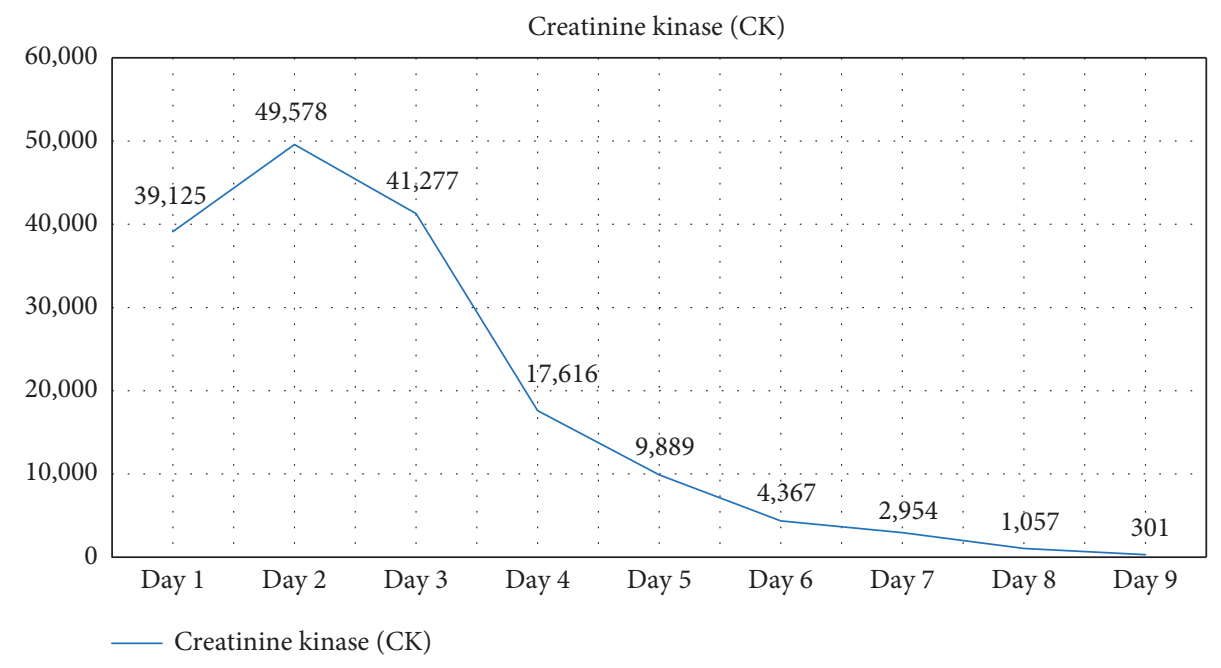

Figure 2: Creatinine kinase trend during hospital stay.

TABLE 1: Reported cases of rhabdomyolysis in adults with Mycoplasma pneumoniae infection.

\begin{tabular}{|c|c|c|c|c|c|}
\hline Reported cases & Date reported & Age/gender & Pneumonia & Creatinine kinase & Other extrapulmonary symptoms \\
\hline Rothstein and Kenney [20] & 1979 & $28 / \mathrm{F}$ & Present & $>40,000$ & Neurological \\
\hline Decaux et al. [21] & 1980 & $60 / \mathrm{M}$ & Absent & 2,900 & Neurological \\
\hline Daxbock et al. [22] & 2002 & $55 / \mathrm{M}$ & Present & 792 & Dermatological \\
\hline Gupta et al. [23] & 2005 & $25 / \mathrm{F}$ & Present & 77,700 & Neurological \\
\hline Khan et al. [24] & 2013 & $37 / \mathrm{M}$ & Present & 14,220 & - \\
\hline Sertogullarindan et al. [25] & 2013 & $25 / \mathrm{F}$ & Present & 51,226 & - \\
\hline Gosselt et al. [26] & 2017 & $47 / \mathrm{M}$ & Present & 25,422 & Dermatological \\
\hline Current case & 2018 & $30 / \mathrm{M}$ & Absent & 49,478 & Gastrointestinal \\
\hline
\end{tabular}

besides ours, where a 60-year-old male presented with rhabdomyolysis and acute encephalitis secondary to $M y$ coplasma pneumoniae but without pneumonia [21]. However, the degree of rhabdomyolysis in our case was much more severe. Our case had a CK of 49,578 at its height, compared to the Decaux et al.'s case that had a CK of 2,900 [21]. The reported cases of rhabdomyolysis with mycoplasma (Table 1) revealed that the severity of rhabdomyolysis varies from case to case. Furthermore, additional extrapulmonary manifestations, besides rhabdomyolysis, were present in some of the cases as well.

The exact mechanism for rhabdomyolysis in $\mathrm{Myco}$ plasma pneumoniae is unclear, but the cases reported suggest a definite association. Mechanisms that have been proposed to cause extrapulmonary manifestations of mycoplasma include direct inflammatory cytokines, indirect autoimmunity or immune complexes, and occlusion of blood vessels causing damage $[8,27]$. It has been suggested that TNF-alpha may be the cause of rhabdomyolysis in mycoplasma although this has not been confirmed yet [28].

The diagnosis of rhabdomyolysis is usually suggested by myalgia or a urinalysis that tests positive for blood in the absence of red blood cells on microscopy. Rhabdomyolysis is formally diagnosed with a CK level 5 times the upper limit of normal and is usually managed with aggressive intravenous hydration to prevent myoglobin-induced renal failure. In general, $50 \%$ of patients who develop rhabdomyolysis also develop renal failure [4, 9]. Diagnosis of Mycoplasma pneumoniae infection, on the other hand, is more difficult to suspect due to the absence of radiographic evidence of pneumonia in some cases. In addition, these patients may not even present with respiratory tract symptoms as seen with our patient. In this case, the patient presented with fever, myalgia, and abdominal complaints, which are not pathognomonic of most mycoplasma cases. The most accurate test to confirm the diagnosis of Mycoplasma pneumoniae infection is a throat culture. However, this is not typically done, as it takes 2-3 weeks to obtain the results. Because of the element of time, polymerase chain reaction (PCR) is typically done when it is available, but most often serology for mycoplasma immunoglobulin $\mathrm{M}$ is performed. Treatment of Mycoplasma pneumoniae infection includes antibiotics such as macrolides, fluoroquinolones, or doxycycline, which is what our patient responded to.

\section{Conclusion}

Traditionally, Mycoplasma pneumoniae is a common cause of atypical community-acquired pneumonia, and many extrapulmonary manifestations have been described. Rhabdomyolysis, specifically, is rarely reported in adults with mycoplasma, especially when no radiographic evidence of pneumonia is present. In this case, there were no typical suggestive complaints for mycoplasma infection, suggesting that rhabdomyolysis secondary to mycoplasma might be underdiagnosed and go untreated. Even though rhabdomyolysis is a rare 
extrapulmonary manifestation of Mycoplasma pneumoniae infection, it should be kept as a differential diagnosis when faced with rhabdomyolysis of unknown etiology despite the absence of pneumonia.

\section{Consent}

Written consent was obtained from the patient to publish his case and case-related imaging.

\section{Conflicts of Interest}

The authors declare that they have no conflicts of interest.

\section{References}

[1] H. M. Foy, G. E. Kenny, M. K. Cooney, and I. D. Allan, "Longterm epidemiology of infections with Mycoplasma pneumoniae," Journal of Infectious Diseases, vol. 139, no. 6, pp. 681-687, 1979.

[2] T. Saraya, "Mycoplasma pneumoniae infection: basics," Journal of General and Family Medicine, vol. 18, no. 3, pp. 118-125, 2017.

[3] B. Abdulhadi and S. S. Bhimji, Pneumonia, Mycoplasma, StatPearls, Treasure Island, FL, USA, 2017.

[4] P. A. Gabow, W. D. Kaehny, and S. P. Kelleher, "The spectrum of rhabdomyolysis," Medicine, vol. 61, no. 3, pp. 141-152, 1982.

[5] N. T. Pesik and E. J. Otten, "Severe rhabdomyolysis following a viral illness: a case report and review of the literature," Journal of Emergency Medicine, vol. 14, no. 4, pp. 425-428, 1996.

[6] J. V. O'Connor and S. K. Iyer, "Myoglobinuria associated with parainfluenza type 2 infection," New York State Journal of Medicine, vol. 82, no. 10, pp. 1469-1470, 1982.

[7] K. Ueda, D. A. Robbins, K. Litaka, and C. C. Linnemann, "Fatal rhabdomyolysis associated with parainfluenza type 3 infection," Hiroshima Journal of Medical Sciences, vol. 27, no. 2, pp. 99-103, 1978.

[8] M. Narita, "Pathogenesis of extrapulmonary manifestations of Mycoplasma pneumoniae infection with special reference to pneumonia," Journal of Infection and Chemotherapy, vol. 16, no. 3, pp. 162-169, 2010.

[9] J. R. Blanco, M. Zabalza, J. Salcedo, L. Echeverria, A. García, and M. Vallejo, "Rhabdomyolysis of infectious and noninfectious causes," Southern Medical Journal, vol. 95, no. 5, pp. 542-544, 2002.

[10] H. M. Heller, S. R. Telford, and J. A. Branda, "Case records of the Massachusetts General Hospital. Case 10-2005. A 73-yearold man with weakness and pain in the legs," New England Journal of Medicine, vol. 352, no. 13, pp. 1358-1364, 2005.

[11] J. P. Knochel and G. E. Moore, "Rhabdomyolysis in Malaria," New England Journal of Medicine, vol. 329, no. 16, pp. 1206-1207, 1993.

[12] U. Singh and W. M. Scheld, "Infectious etiologies of rhabdomyolysis: three case reports and review," Clinical Infectious Diseases, vol. 22, no. 4, pp. 642-649, 1996.

[13] H. Osamah, R. Finkelstein, and J. G. Brook, "Rhabdomyolysis complicating acute Epstein-Barr virus infection," Infection, vol. 23, no. 2, pp. 119-120, 1995.

[14] M. J. Will, R. B. Hecker, and P. I. Wathen, "Primary varicellazoster-induced rhabdomyolysis," Southern Medical Journal, vol. 89, no. 9, pp. 915-920, 1996.
[15] A. Shah, F. Check, S. Baskin, T. Reyman, and R. Menard, "Legionnaires' disease and acute renal failure: case report and review," Clinical Infectious Diseases, vol. 14, no. 1, pp. 204207, 1992.

[16] V. Spataro and C. Marone, "Rhabdomyolysis associated with bacteremia due to Streptococcus pneumoniae: case report and review," Clinical Infectious Diseases, vol. 17, no. 6, pp. 1063-1064, 1993.

[17] G. F. Falasca and A. J. Reginato, "The spectrum of myositis and rhabdomyolysis associated with bacterial infection," Journal of Rheumatology, vol. 21, no. 10, pp. 1932-1937, 1994.

[18] S. Kawamura, H. Ikematsu, and H. Ogimoto, "Two cases of psittacosis accompanied with rhabdomyolysis," Kansenshogaku Zasshi, vol. 64, no. 9, pp. 1239-1243, 1990.

[19] O. J. Rheingold, "Myoglobinuria and renal failure associated with typhoid fever," JAMA, vol. 238, no. 4, p. 341, 1977.

[20] T. L. Rothstein and G. E. Kenny, "Cranial neuropathy, myeloradiculopathy, and myositis: complications of $\mathrm{Myco-}$ plasma pneumoniae infection," Archives of Neurology, vol. 36, no. 8, pp. 476-477, 1979.

[21] G. Decaux, M. Szyper, M. Ectors, A. Cornil, and L. Franken, "Central nervous system complications of Mycoplasma pneumoniae," Journal of Neurology, Neurosurgery and Psychiatry, vol. 43, no. 10, pp. 883-887, 1980.

[22] F. Daxbock, G. Brunner, H. Popper et al., "A case of lung transplantation following Mycoplasma pneumoniae infection," European Journal of Clinical Microbiology and Infectious Diseases, vol. 21, no. 4, pp. 318-322, 2002.

[23] R. Gupta, A. Gupta, V. Goyal, R. Guleria, and A. Kumar, "Mycoplasma pneumonia associated with rhabdomyolysis and the Guillain-Barre syndrome," Indian Journal of Chest Diseases and Allied Sciences, vol. 47, no. 4, pp. 305-308, 2005.

[24] F. Y. Khan and H. Sayed, "Rhabdomyolysis associated with Mycoplasma pneumoniae pneumonia," Hong Kong Medical Journal, vol. 18, no. 3, pp. 247-249, 2012.

[25] B. Sertogullarindan, M. B. Ozbay, F. U. Ertem et al., "Rhabdomyolysis associated with Mycoplasma pneumoniae infection," Polskie Archiwum Medycyny Wewnetrznej, vol. 123, no. 1-2, pp. 66-67, 2013.

[26] A. Gosselt, J. Olijhoek, and T. Wierema, "Severe asymptomatic rhabdomyolysis complicating a Mycoplasma pneumonia," BMJ Case Reports, vol. 2017, 2017.

[27] M. Narita, "Classification of extrapulmonary manifestations due to Mycoplasma pneumoniae infection on the basis of possible pathogenesis," Frontiers in Microbiology, vol. 7, p. 23, 2016.

[28] T. Oishi, M. Narita, H. Ohya et al., "Rhabdomyolysis associated with antimicrobial drug-resistant Mycoplasma pneumoniae," Emerging Infectious Diseases, vol. 18, no. 5, pp. $849-851,2012$. 


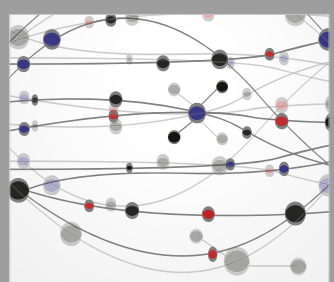

The Scientific World Journal
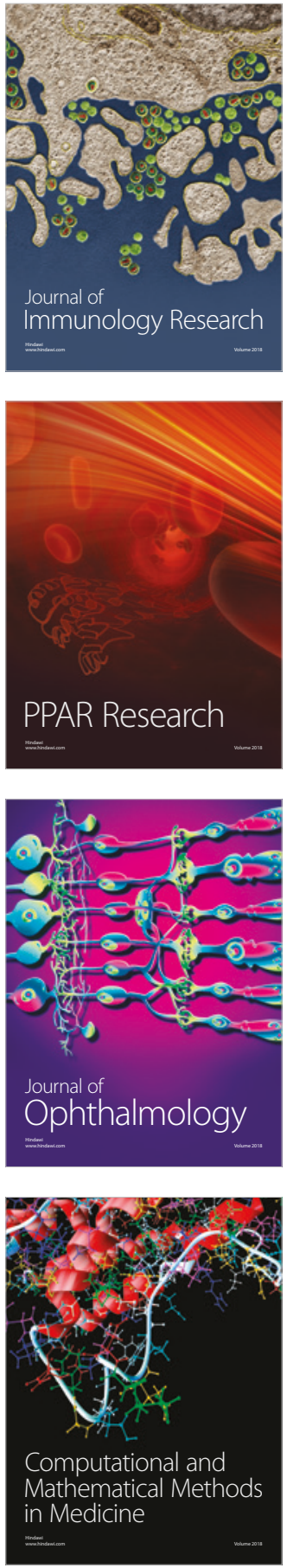

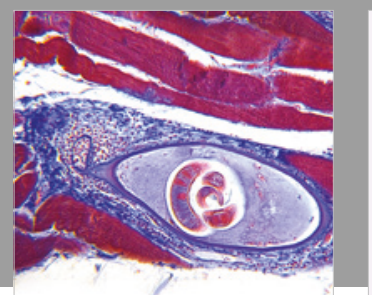

Gastroenterology Research and Practice

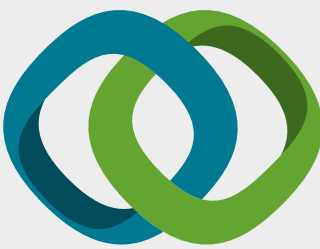

\section{Hindawi}

Submit your manuscripts at

www.hindawi.com
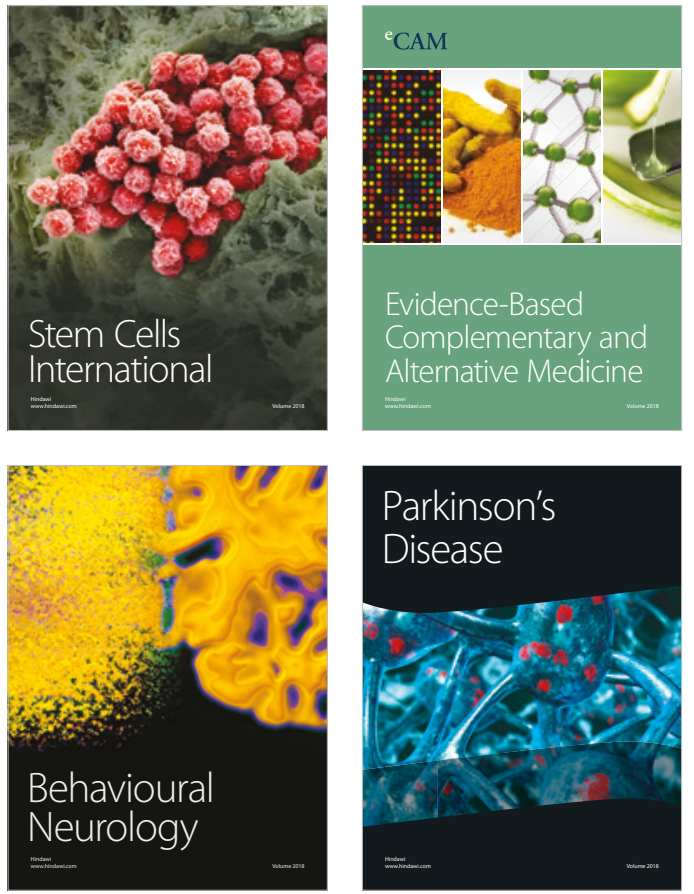

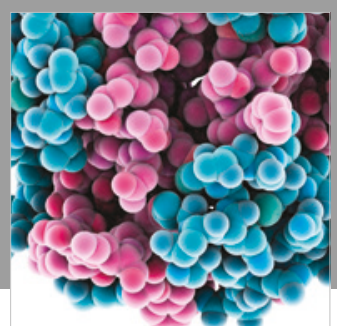

ournal of

Diabetes Research

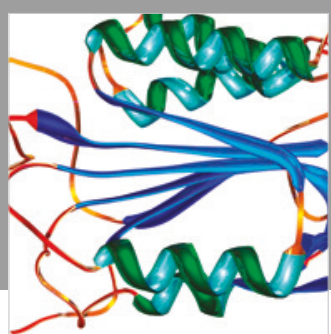

Disease Markers
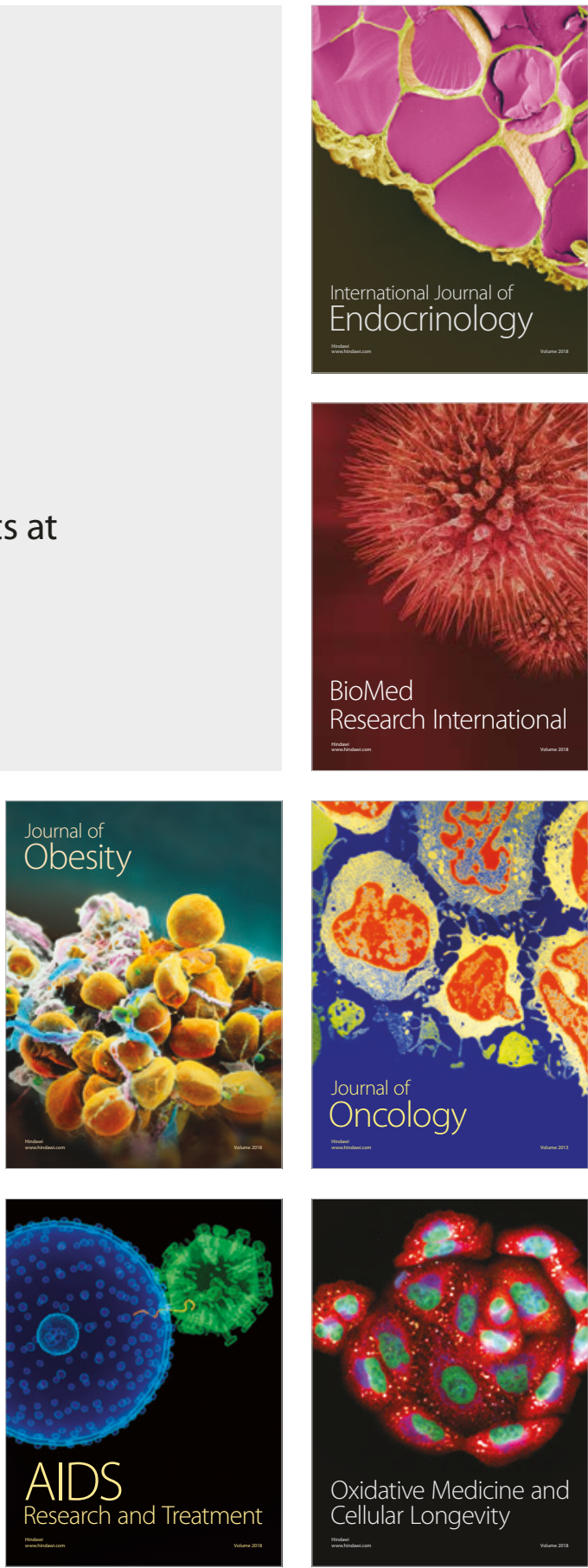